\title{
Time Domain Beam Propagation Method for the simulation of temporal solitons in periodic media
}

\author{
F. Horst, H.J.W.M. Hoekstra, A. Driessen, Th.J.A. Popma \\ MESA Research Institute, Lightwave Devices Group, University of Twente, P.O. Box 217, NL- \\ 7500 AE Enschede, The Netherlands. Tel.: X-31-53-892719, Fax.: X-31-53-309547. E-mail: \\ F.Horst@el.utwente.nl.
}

\begin{abstract}
:
A Time Domain Beam Propagation Method for the simulation of optical pulses propagating through Kerr-nonlinear structures is presented. The method is verified by simulation of the known solitary wave solutions in nonlinear periodic media.
\end{abstract}

\section{Introduction}

A Time Domain Beam Propagation Method (TD-BPM) has been implemented to simulate the propagation of short optical pulses through a one-dimensional nonlinear periodic structure. It has been shown by a coupled mode model of the non-linear periodic structure $[1,2]$ that these pulses exhibit soliton-like behavior. This model, an extended version of the massive Thirring model, yields soliton-like solutions which are characterized by two parameters $Q$ and $v$ which determine pulse duration and velocity of the optical pulse.

Modeling and simulation by coupled mode models is limited to systems with a weak interaction between the modes. Also some structures, for example chirped periodic media, are hard to implement in coupled mode models. The TD-BPM allows a wider range of (periodic and nonperiodic) structures. The structure that is used in the simulation is described by the distribution of the index of refraction (linear and nonlinear, real and imaginary). This description is more easily connected to the physical implementation of the structure than the description by coupling constants as is used by the coupled mode method. Therefore the TD-BPM method can be used as a realistic design tool.

\section{Theory}

The TD-BPM is based on the scalar waveequation for a two-dimensional space, described by one spatial coordinate $z$ and the temporal coordinate $t$. The electrical field is split into a carrier wave oscillating at frequency $\omega_{0}$, and a slowly varying envelope: $E(z, t)=\Psi(z, t) e^{-i \omega_{0} t}$. By insertion of this equation into the wave equation and neglecting the small second derivative to time of the slowly varying envelope, a propagation equation for the envelope of the electrical field is found:

$$
\frac{\partial \Psi}{\partial t}=\frac{1}{2} i\left\{\frac{c_{0}^{2}}{n^{2} \omega_{0}} \frac{\partial^{2} \Psi}{\partial z^{2}}+\omega_{0} \Psi\right\}
$$

It should be noted that only the highly oscillatory terms along the temporal axis have been eliminated from $\Psi(z, t)$. The harmonical carrier along the $z$-axis is still present in the envelope function. The geometry of the structure is defined by the index of refraction: $n(z, t)=$ $n_{0}(z)+n_{2}(z)|E(z, t)|^{2}$. Both $n_{0}(z)$ and $n_{2}(z)$ may have imaginary contributions.

Using a standard Crank-Nicholson approach, the propagation equation is discretised onto a rectangular grid, indexed by the grid coordinates $s$ and $i$ along the temporal and spatial axes respectively:

$$
\begin{gathered}
\Psi_{i-1}^{s+1}+(-2-p+q) \Psi_{i}^{s+1}+\Psi_{i+1}^{s+1}= \\
-\Psi_{i-1}^{s}-(-2+p+q) \Psi_{i}^{s}-\Psi_{i+1}^{s}
\end{gathered}
$$

with

$$
p=-4 i \frac{\left(n_{i}^{s}\right)^{2} \omega_{0}}{c_{0}^{2}} \frac{\Delta z^{2}}{\Delta t}, \quad q=\frac{\left(n_{i}^{s}\right)^{2} \omega_{0}^{2}}{c_{0}^{2}} \Delta z^{2}
$$

At the upper and lower spatial boundaries of the simulation space a variant of the 'Transparent Boundary Conditions' [3] is implemented. 


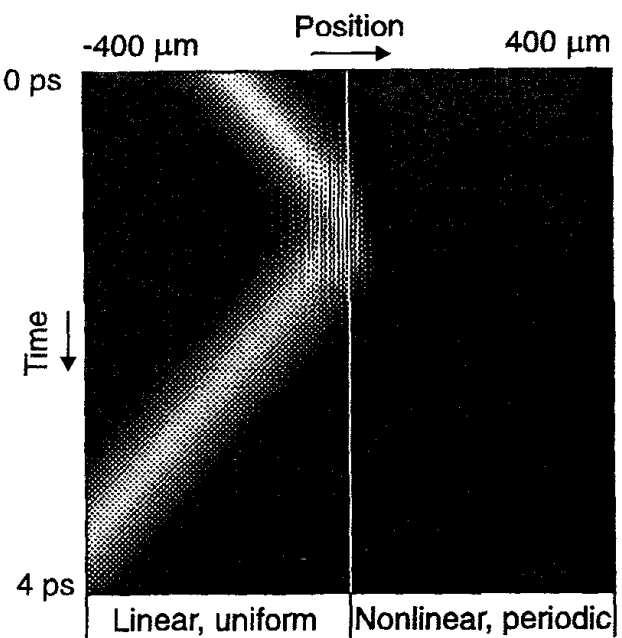

Figure 1: Reflection of low-intensity input pulse on nonlinear Bragg reflector.

\section{Verification and Demonstration}

The TD-BPM simulation algorithm has been verified by defining a nonlinear periodic structure in the simulation space and starting at $t=0$ one of the corresponding solitary wave solutions of the extended Thirring model $[1,2]$. All combinations of $v$ and $Q$ parameters that were tried resulted in stable pulses propagating at the re quired speed.

An example of a simulation of soliton selfswitching is given in figures 1 and 2 . In these simulations the left half of the simulation space consists of a uniform, linear medium where $n_{0}=$ 1.5. In the right half of the simulation space the index of refraction is modulated by $\delta n=$ $0.015 \cdot \sin (2 \pi z / \Lambda), \Lambda=300 \mathrm{~nm}$. Also a nonlinearity of $n_{2}=5 \cdot 10^{-18} \mathrm{~m}^{2} / W$ is added. The peak-powers of the input pulses for the low- and high-intensity simulations are $8 \cdot 10^{14} \mathrm{~W} / \mathrm{m}^{2}$ and $11 \cdot 10^{14} \mathrm{~W} / \mathrm{m}^{2}$ respectively.

\section{Conclusions}

The TD-BPM simulation algorithm offers a good method for the simulation of soliton propagation through periodic nonlinear media. Compared to coupled mode simulation algorithms, the TDBPM may be used to simulate a wider range of

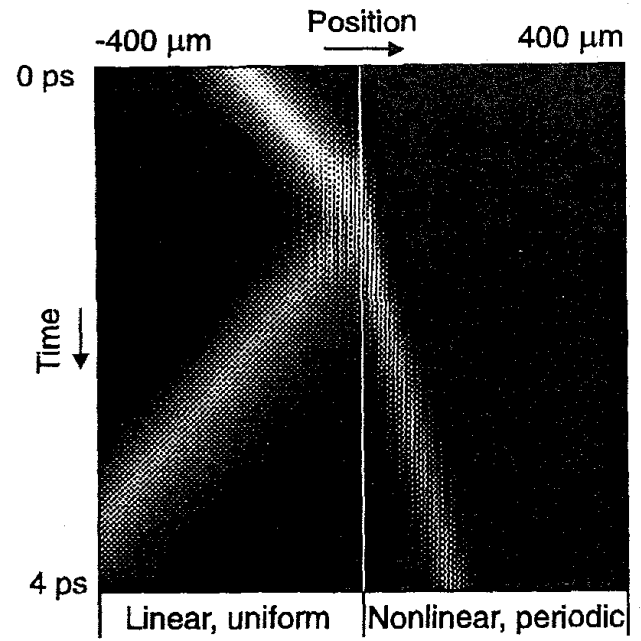

Figure 2: Soliton transmission of high-intensity input pulse through nonlinear Bragg reflector.

structures and uses a description of the structure that is easily derived from the actual physical implementation of the structure. The algorithm can be used to simulate the propagation of short optical pulses through arbitrary onedimensional structures. This includes soliton propagation through grating structures (chirped, varying grating depth), but also propagation of short optical pulses through e.g. nonlinear Fabry-Perot interferometers. Due to the density of the discretisation points needed, the use of the algorithm on workstations is, for the time being, limited to structures with a maximum length of a few millimeters.

\section{References}

[1] C. Martijn de Sterke, J.E. Sipe, Phys. Rev. A, vol 42, no. 1, pp. 550-555, 1990.

[2] A.B. Aceves, S. Wabnitz, Phys. Lett. A, vol. 141, no. 1, pp. 37-42, 1989.

[3] G.R. Hadley, Opt. Lett., vol 16, no. 9, pp. 624-626, 1991 . 